\title{
Single kick approximations for beam-beam deflections
}

\author{
Takahiko Koyama \\ Laboratory of Nuclear Studies, Cornell University, Ithaca, New York 14853
}

(Received 19 November 1998; published 22 February 1999)

\begin{abstract}
A six-dimensional symplectic beam-beam interaction map using finite discrete slices of a strong beam is extended to infinitesimal slices. The new map is calculated under the assumption of a longitudinal Gaussian distribution with approximations. A round Gaussian beam is simulated to demonstrate accuracies of the approximations. [S1098-4402(99)00028-2]

PACS numbers: $29.27 . \mathrm{Bd}$
\end{abstract}

\section{INTRODUCTION}

In early beam-beam simulations the dependence on longitudinal position has been ignored in calculating the beam-beam force exerted on a particle. The kick is calculated as if the particle is located at the center of the bunch and collides with all of the opposing bunch at the interaction point (IP). As a result of this simplified approach, the strengths of kicks are the same for a particle in the middle of the bunch and the tail of the bunch as long as those two particles have the same transverse coordinate. Clearly, this approach has only limited validity.

For today's high luminosity storage ring, where $\beta$ can be very small at the IP, this simplified approach must be replaced by a method which includes longitudinal effects [1]. Such longitudinal effects can be divided into two parts: the transverse kick should be influenced by the longitudinal position of the particle and there should be a longitudinal kick appearing as an energy change.

First, we will review a synchro-beam map developed by Hirata et al. In this introductory section we emphasize the physics of beam-beam interaction rather than the purely mathematical manipulation of formulas using Hamiltonian formulation.

Then, we will apply the map to infinitesimal strong slices of a longitudinally Gaussian distributed strong beam. With proper approximation, we will make dramatic simplification of the formulas.

As an application of the general theory developed here, we will further customize the method to round Gaussian beams. The integrations over infinitesimal slices are carried out with approximations and a point interaction formula is obtained.

Finally, to test the accuracy of our result, a simulation has been carried out. Comparisons with discrete slice methods using the synchro-beam map suggested by Hirata et al. are made with various levels of approximations developed in this paper.

\section{A. Synchro-beam map by Hirata et al.}

Here, we will review the method called synchro-beam map, developed by Hirata et al. [2]. Before we go any farther, we should state clearly that throughout this paper we use weak-strong formalism. Thus, the opposing strong beam is assumed to be unaffected by the interaction.

Suppose that there is a test particle located at $\left(x, p_{x}, y, p_{y}, z, \epsilon\right)$, where $p_{x}=d x / d s, \quad p_{y}=d y / d s$, $\epsilon=p / p_{0}$, and a strong slice is located at $z_{*}$ when the centers of both bunches are at IP. As can be seen from Fig. 1, in general, the test particle and the slice do not collide at this time. In this particular case, they will collide some time later. After $\delta t=\left(z-z_{*}\right) / 2 c$ (seen in Fig. 2), they will collide at a collision point (CP). Ignoring synchrotron oscillations of the particle temporarily ${ }^{1}$ (i.e., the particle moves at uniform speed $c$ in the longitudinal direction), the transverse coordinates of the test particle at the $\mathrm{CP}$ will be

$$
\begin{aligned}
X & =x+p_{x} S, \\
P_{X} & =p_{x}, \\
Y & =y+p_{y} S, \\
P_{Y} & =p_{y},
\end{aligned}
$$

where

$$
S=\frac{z-z_{*}}{2} .
$$

Note that in the synchro-beam interaction, the size (or shape) of the strong bunch also changes according to the Twiss parameters at IP.

It is easy to verify that the transformation in Eq. (1) is induced by

$$
\begin{aligned}
\mathbf{X} & =\exp \left(:-\frac{p_{x}^{2}+p_{y}^{2}}{2} S:\right) \mathbf{x} \\
& \equiv \exp \left(:-D\left(S\left(z, z_{*}\right)\right):\right) \mathbf{x},
\end{aligned}
$$

where a new notation : $D: \mathbf{x}$ is a Poisson bracket $[D, \mathbf{x}]$. Since this transformation is generated by continuous Lie transformation, it is guaranteed to be canonical. We

\footnotetext{
${ }^{1}$ This is a good approximation since the synchrotron oscillation is slower than the time scale we are dealing with for beam-beam interaction.
} 


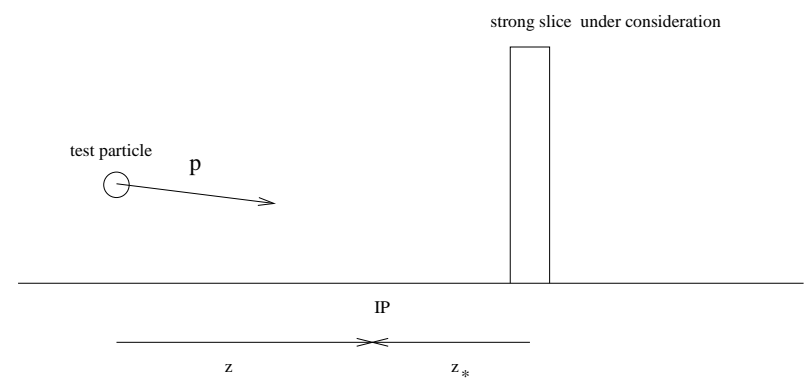

FIG. 1. A test particle and a strong slice under consideration when the center of the bunch and its opposing bunch are at IP. In this figure, suppose that the bunch containing the test particle advances to the right.

shall obtain the new longitudinal coordinate under this transformation,

$$
Z=z-[D, z]+\frac{1}{2}[D,[D, z]]+\cdots .
$$

The Poisson bracket $[D, z]$ is easily calculated as

$$
\left[\frac{\left(p_{x}^{2}+p_{y}^{2}\right) S}{2}, z\right]=0 .
$$

Thus, the second and higher orders vanish.

In the same manner,

$$
\begin{aligned}
P_{Z}=\epsilon-[D, \epsilon]+\frac{1}{2}[D,[D, \epsilon]]+\cdots, & \\
{[D, \epsilon] } & =\left[\frac{\left(p_{x}^{2}+p_{y}^{2}\right) S}{2}, \epsilon\right] \\
& =\frac{\partial}{\partial z} \frac{\left(p_{x}^{2}+p_{y}^{2}\right) S}{2} \\
& =\frac{p_{x}^{2}+p_{y}^{2}}{4}
\end{aligned}
$$

The second-order term is

$$
[D,[D, \epsilon]]=\left[\frac{\left(p_{x}^{2}+p_{y}^{2}\right) S}{2}, \frac{p_{x}^{2}+p_{y}^{2}}{4}\right]=0,
$$

and higher-order terms are identically 0 . Under the transformation, the longitudinal coordinates are modi-

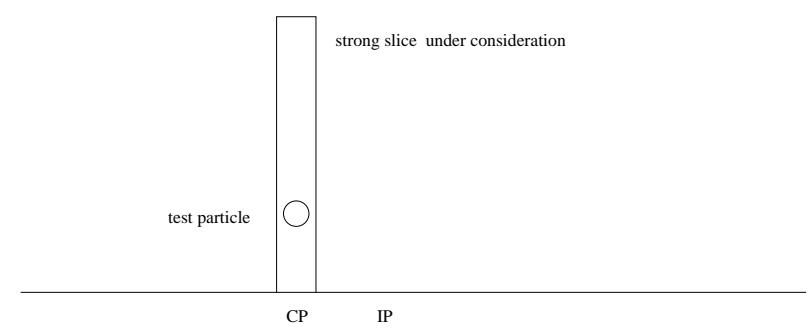

FIG. 2. A test particle and a strong slice under collision at CP. fied to

$$
\begin{aligned}
Z & =z \\
P_{Z} & =\epsilon-\frac{p_{x}^{2}+p_{y}^{2}}{4} .
\end{aligned}
$$

Hirata et al. proposed a synchro-beam mapping for a particle-slice interaction, which is generated by

$H_{b b}\left(x, p_{x}, y, p_{y}, z, \epsilon ; s\right)=n_{*} U\left[X, Y ; Q_{*}\left(S\left(Z, z_{*}\right)\right)\right] \delta(s)$,

where $n_{*}$ is the number of particles in the slice, $Q_{*}$ represents a set of auxiliary parameters characterizing the transverse distribution function of the strong beam, and $U$ is the electric scalar potential of the strong slice. In particular, for bi-Gaussian, $Q_{*}$ is just $\sigma_{x}$ and $\sigma_{y}$. To reflect the change in the strong beam due to translation of CP, $Q_{*}$ is a function of the displacement $S$.

Then, using this map, we can apply the beam-beam interaction based on the coordinate at the $\mathrm{CP}$,

$$
X^{\text {new }}=\exp \left(:-n_{*} U\left[X, Y ; Q_{*}\left(S\left(Z, z_{*}\right)\right)\right]:\right) X .
$$

It is obvious that the spatial coordinates $X, Y$, and $Z$ remain unchanged under the transformation due to the commutations to the operator $U\left[X, Y ; Q_{*}\left(S\left(Z, z_{*}\right)\right)\right]$. However, the momenta are changed.

Let us define

$$
\begin{aligned}
& \exp \left[-: n_{*} U(X, Y ; Z):\right]\left(\begin{array}{c}
P_{X} \\
P_{Y} \\
P_{Z}
\end{array}\right) \\
& \equiv\left(\begin{array}{c}
P_{X}-n_{*} f_{X}(X, Y ; Z) \\
P_{Y}-n_{*} f_{Y}(X, Y ; Z) \\
P_{Z}-n_{*} g(X, Y ; Z)
\end{array}\right),
\end{aligned}
$$

where, following Hirata et al., we have defined

$$
\begin{aligned}
f_{X}(X, Y ; Z) & \equiv \frac{\partial}{\partial X} U(X, Y ; Z), \\
f_{Y}(X, Y ; Z) & \equiv \frac{\partial}{\partial Y} U(X, Y ; Z), \\
g(X, Y ; Z) & \equiv \frac{\partial}{\partial Z} U(X, Y ; Z) .
\end{aligned}
$$

It is clear that the second or higher order of : $U$ : does not contribute since the $f$ 's and $g$ are functions of $X, Y$, and $Z$ only.

Since $U$ depends on $Z$ implicitly, we need to take special care to obtain $g$ :

$$
\begin{aligned}
g(X, Y ; Z) & =g\left[X, Y, Q_{*}\left(S\left(Z, z_{*}\right)\right)\right] \\
& =\frac{\partial U}{\partial Q} \frac{d Q}{d S} \frac{\partial S}{\partial Z}=\frac{1}{2} \frac{\partial U}{\partial Q} \frac{d Q}{d S} .
\end{aligned}
$$

For the last equality, we have used Eq. (2). 
After we apply the kicks, we transfer the test particle back to the original $z$. We summarize the final result of synchro-beam interaction by a single slice below:

$$
\begin{aligned}
x^{\text {new }}= & x+n_{*} S\left(z, z_{*}\right) f_{X}(X, Y ; Z), \quad p_{x}^{\text {new }}=p_{x}-n_{*} f_{X}(X, Y ; Z), \\
y^{\text {new }}= & y+n_{*} S\left(z, z_{*}\right) f_{Y}(X, Y ; Z), \quad p_{y}^{\text {new }}=p_{y}-n_{*} f_{Y}(X, Y ; Z), \quad z^{\text {new }}=z, \\
\epsilon^{\text {new }}= & \epsilon-\frac{1}{2} n_{*} f_{X}(X, Y ; Z)\left[p_{x}-\frac{n_{*}}{2} f_{X}(X, Y ; Z)\right] \\
& -\frac{1}{2} n_{*} f_{Y}(X, Y ; Z)\left[p_{y}-\frac{n_{*}}{2} f_{Y}(X, Y ; Z)\right]-n_{*} g(Z, Y ; Z) .
\end{aligned}
$$

The same calculation is performed slice after slice to obtain the total effect due to the strong bunch.

\section{B. Synchro-beam map applied to infinitesimal slices}

Here, the synchro-beam map is applied to a discrete number of slices. Suppose there are $L$ discrete slices of opposing bunch. Thus, we apply Eq. (11) $L$ times in the order opposite to the physical order;

$$
\begin{aligned}
\mathbf{x}^{\text {new }} & =\exp \left(-n_{* 1}: U_{1}:\right) \exp \left(-n_{* 2}: U_{2}:\right) \cdots \exp \left(-n_{* L}: U_{L}:\right) \mathbf{x} \\
& =\left(1-n_{* 1}: U_{1}:+\cdots\right)\left(1-n_{* 2}: U_{2}:+\cdots\right) \cdots\left(1-n_{* L}: U_{L}:+\cdots\right) \mathbf{x} \\
& \simeq\left(1-\sum_{k=1}^{L} n_{* k}: U_{k}:+\frac{1}{2} n_{* k}^{2}: U_{k}:^{2}+\sum_{k=1}^{L} \sum_{l>k}^{L} n_{* k} n_{* l}: U_{k}:: U_{l}:\right) \mathbf{x},
\end{aligned}
$$

where $\mathbf{x}$ represents $\left(x, y, z, p_{x}, p_{y}, \boldsymbol{\epsilon}\right)$.

The second-order term, such as : $U_{k}:: U_{l}: x$, contains $f_{x}$ twice. Typically,

$$
f_{x} \approx \frac{r_{e}}{\gamma_{0} \sigma_{x}} .
$$

This is a small dimensionless value, even after multiplication by the total number of particles in a strong bunch $N$. As a good approximation, we will ignore the second or higher terms. Thus, an incoming particle is influenced by strong slices directly and independently. Then, the expression simplifies to

$$
\mathbf{x}^{\text {new }}=\left(1-\sum_{k=1}^{L} n_{* k}: U_{k}:\right) \mathbf{x} .
$$

The slices considered in the previous subsection are made infinitesimal and integrated to obtain the effects given by the entire opposing bunch.

Assuming that the opposing beam is Gaussian distributed in longitudinal direction, we can convert the summation into an integration as

$$
\sum_{k=1}^{L} n_{* k} \Rightarrow N \int_{-\infty}^{\infty} \frac{d z_{*}}{\sqrt{2 \pi \sigma_{z}^{2}}} \exp \left(-\frac{z_{*}^{2}}{2 \sigma_{z}^{2}}\right) .
$$

Using this expression, Eq. (18) can be converted to

$$
\begin{aligned}
\mathbf{x}^{\text {new }}= & \mathbf{x}-N \int_{-\infty}^{\infty} \frac{d z_{*}}{\sqrt{2 \pi \sigma_{z}^{2}}} \\
& \times \exp \left(-\frac{z_{*}^{2}}{2 \sigma_{z}^{2}}\right)\left[U\left(X, Y ; Q\left(Z, z_{*}\right)\right), \mathbf{x}\right] .
\end{aligned}
$$

This simple-looking equation is the core of this entire paper, and all following sections are devoted to instances of its evaluation. However, this equation cannot be evaluated easily. Instead, we transform $\mathbf{x}$ into $\mathbf{X}$ first and apply the beam-beam interaction,

$$
\begin{aligned}
\mathbf{X}^{\text {new }}= & \mathbf{X}-N \int_{-\infty}^{\infty} \frac{d z_{*}}{\sqrt{2 \pi \sigma_{z}^{2}}} \\
& \times \exp \left(-\frac{z_{*}^{2}}{2 \sigma_{z}^{2}}\right)\left[U\left(X, Y ; Q\left(Z, z_{*}\right)\right), \mathbf{X}\right] .
\end{aligned}
$$

Then, we transform $\mathbf{X}^{\text {new }}$ back to the IP to obtain $\mathbf{x}^{\text {new }}$.

\section{APPLICATION TO ROUND GAUSSIAN BEAM}

\section{A. Introduction}

Now, we assume that a bunch and its opposing strong bunch are round and have transverse Gaussian distributions. Then, the "potential" of the beam can be expressed [3] as

$$
U(R, S)=-\frac{r_{e}}{\gamma_{0}} \int_{0}^{\infty} d u \frac{\exp \left(\frac{-R}{2 \Sigma_{11}(S)+u}\right)}{2 \Sigma_{11}(S)+u},
$$

where $\Sigma_{11}(S)$ is the adjusted beam size at the corresponding CP. Ignoring the effects of beam-beam interaction, the region around IP is treated as drift space. Thus,

$$
\beta(s)=\beta_{0}-2 \alpha_{0} s+\gamma_{0} s^{2},
$$

where $\alpha_{0}, \beta_{0}$, and $\gamma_{0}$ are Twiss parameters at the IP. To shorten the writing, we drop the subscript 0 in further discussion. And, to avoid the confusion between $\beta(s)$ and $\beta_{0}$, we explicitly address $\beta(s)$. Then, it is a good 
approximation to take

$$
\Sigma_{11}(s)=\epsilon_{0} \beta(s)=\epsilon_{0}\left(\beta-2 \alpha s+\gamma s^{2}\right) .
$$

To obtain $f$ or $g$ we use Eq. (13),

$$
f=\frac{\partial U}{\partial R}=\frac{r_{e}}{\gamma_{0}} \int_{0}^{\infty} d u \frac{2 R}{\left[2 \Sigma_{11}(S)+u\right]^{2}} \exp \left(\frac{-R^{2}}{2 \Sigma_{11}(S)+u}\right) .
$$

To integrate this, we change the variable $u$ to

$$
v=\frac{1}{2 \Sigma_{11}(S)+u} .
$$

Then,

$$
f=-\frac{2 R r_{e}}{\gamma_{0}} \int_{1 / 2 \Sigma_{11}}^{0} d v \exp \left(-R^{2} v\right)=-\frac{2 r_{e}}{\gamma_{0} R}\left[\exp \left(\frac{-R^{2}}{2 \Sigma_{11}}\right)-1\right]
$$

In the same manner,

$$
\begin{aligned}
g & =\frac{1}{2} \frac{d \Sigma_{11}}{d S} \frac{\partial U}{\partial \Sigma_{11}} \\
& =-\frac{r_{e}}{\gamma_{0}} \frac{1}{2} \frac{d \Sigma_{11}}{d S} \int_{0}^{\infty} d u \frac{\partial}{\partial \Sigma_{11}} \frac{\exp \left(\frac{-R^{2}}{2 \Sigma_{11}(S)+u}\right)}{2 \Sigma_{11}(S)+u} \\
& =-\frac{r_{e}}{2 \gamma_{0}} \frac{d \Sigma_{11}}{d S} 2 \int_{0}^{\infty} d u\left(\frac{r^{2}}{\left(2 \Sigma_{11}+u\right)^{3}}-\frac{1}{\left(2 \Sigma_{11}+u\right)^{2}}\right) \exp \left(\frac{-R^{2}}{2 \Sigma_{11}(S)+u}\right) \\
& =-\frac{r_{e}}{\gamma_{0}} \frac{d \Sigma_{11}}{d S} \int_{1 / 2 \Sigma_{11}}^{0} d v\left(1-R^{2} v\right) e^{-v R^{2}} \\
& =\frac{r_{e}}{\gamma_{0}} \frac{d \Sigma_{11}}{d S} \frac{1}{2 \Sigma_{11}} e^{-\left(r^{2} / 2 \Sigma_{11}\right)} .
\end{aligned}
$$

Incorporating this into Eq. (21),

$$
P_{R}^{\text {new }}=r-N \int_{-\infty}^{\infty} \frac{d z_{*}}{\sqrt{2 \pi \sigma_{z}^{2}}} \exp \left(-\frac{z_{*}^{2}}{2 \sigma_{z}^{2}}\right)\left[U, P_{R}\right] .
$$

Since the Poisson bracket

$$
\begin{gathered}
{\left[U, P_{R}\right]=-\frac{\partial U}{\partial R} \equiv f(R, S)} \\
P_{R}^{\text {new }}=P_{R}-N \int_{-\infty}^{\infty} \frac{d z_{*}}{\sqrt{2 \pi \sigma_{z}^{2}}} \exp \left(-\frac{z_{*}^{2}}{2 \sigma_{z}^{2}}\right) f .
\end{gathered}
$$

Thus, transforming back to the original coordinate, we obtain

$$
p_{r}^{\text {new }}=p_{r}-N \int_{-\infty}^{\infty} \frac{d z_{*}}{\sqrt{2 \pi \sigma_{z}^{2}}} \exp \left(-\frac{z_{*}^{2}}{2 \sigma_{z}^{2}}\right) f \equiv p_{r}-F,
$$

where we defined

$$
F=N \int_{-\infty}^{\infty} \frac{d z_{*}}{\sqrt{2 \pi \sigma_{z}^{2}}} \exp \left(-\frac{z_{*}^{2}}{2 \sigma_{z}^{2}}\right) f
$$

As for the transverse spatial coordinate $r$,

$$
R^{\text {new }}=R-N \int_{-\infty}^{\infty} \frac{d z_{*}}{\sqrt{2 \pi \sigma_{z}^{2}}} \exp \left(-\frac{z_{*}^{2}}{2 \sigma_{z}^{2}}\right)[U(R), R]=R,
$$


as expected. Transforming back to the original coordinate,

$$
r^{\text {new }}=R^{\text {new }}-p_{r}^{\text {new }} S=R-\left[p_{r}-N \int_{-\infty}^{\infty} \frac{d z_{*}}{\sqrt{2 \pi \sigma_{z}^{2}}} \exp \left(-\frac{z_{*}^{2}}{2 \sigma_{z}^{2}}\right) f\right] S .
$$

In terms of the original coordinates,

$$
r^{\text {new }}=r+N \int_{-\infty}^{\infty} \frac{d z_{*}}{\sqrt{2 \pi \sigma_{z}^{2}}} \exp \left(-\frac{z_{*}^{2}}{2 \sigma_{z}^{2}}\right) f S \equiv r+H
$$

where we defined

As for $z$

$$
H=N \int_{-\infty}^{\infty} \frac{d z_{*}}{\sqrt{2 \pi \sigma_{z}^{2}}} \exp \left(-\frac{z_{*}^{2}}{2 \sigma_{z}^{2}}\right) f S
$$

$$
Z^{\text {new }}=Z-N \int_{-\infty}^{\infty} \frac{d z_{*}}{\sqrt{2 \pi \sigma_{z}^{2}}} \exp \left(-\frac{z_{*}^{2}}{2 \sigma_{z}^{2}}\right)[U(R, Z), Z]=Z
$$

Thus,

$$
z^{\text {new }}=Z^{\text {new }}=Z=z \text {. }
$$

Note that this is always true in synchro-beam map. In other words, the longitudinal coordinate is invariant under the synchro-beam map.

Finally,

$$
\begin{gathered}
P_{Z}^{\text {new }}=P_{Z}-N \int_{-\infty}^{\infty} \frac{d z_{*}}{\sqrt{2 \pi \sigma_{z}^{2}}} \exp \left(-\frac{z_{*}^{2}}{2 \sigma_{z}^{2}}\right)\left[U(R, Z), P_{Z}\right], \\
{\left[U, P_{Z}\right]=-\frac{\partial U}{\partial Z}=-\frac{1}{2} \frac{\partial U}{\partial S} \equiv g,} \\
P_{Z}^{\text {new }}=P_{Z}-N \int_{-\infty}^{\infty} \frac{d z_{*}}{\sqrt{2 \pi \sigma_{z}^{2}}} \exp \left(-\frac{z_{*}^{2}}{2 \sigma_{z}^{2}}\right) g \equiv P_{Z}-G,
\end{gathered}
$$

where we defined

$$
G=-N \int_{-\infty}^{\infty} \frac{d z_{*}}{\sqrt{2 \pi \sigma_{z}^{2}}} \exp \left(-\frac{z_{*}^{2}}{2 \sigma_{z}^{2}}\right) g
$$

Now, going back to the original coordinate system,

$$
\begin{aligned}
\epsilon^{\text {new }} & =P_{Z}^{\text {new }}+\frac{p^{\text {new }}}{4}=\left(P_{Z}-G\right)+\frac{\left(p_{r}-F\right)^{2}}{4} \\
& =\left(\epsilon-\frac{p_{r}^{2}}{4}-G\right)+\frac{\left(p_{r}-F\right)^{2}}{4} .
\end{aligned}
$$

This implies

$$
\epsilon^{\text {new }}=\epsilon-G-\frac{1}{2} p_{r} F+\frac{1}{4} F^{2}
$$

\section{B. Detailed computation}

Inserting $r+p_{r} S$ in place of $R$ in Eq. (32) and in place of $f$ in Eq. (27), we obtain an expression

$$
p_{r}^{\text {new }}=p_{r}-\frac{N r_{e}}{\gamma_{0}} \int_{-\infty}^{\infty} \frac{d z_{*}}{\sqrt{2 \pi \sigma_{z}^{2}}} \exp \left(-\frac{z_{*}^{2}}{2 \sigma_{z}^{2}}\right) \frac{2}{r+S p_{r}}\left[1-\exp \left(-\frac{\left(r+S p_{r}\right)^{2}}{2 \Sigma_{11}(S)}\right)\right] .
$$

Now, changing the variable of the integral from $z *$ to $S$, according to Eq. (2),

$$
d z_{*}=-2 d S .
$$


Then,

$$
\begin{aligned}
p_{r}^{\text {new }} & =p_{r}-\frac{N r_{e}}{\gamma_{0}} \int_{-\infty}^{\infty} \frac{d S}{\sqrt{2 \pi \sigma_{z}^{2}}} \exp \left(-\frac{(z-2 S)^{2}}{2 \sigma_{z}^{2}}\right) \frac{4}{r+S p_{r}}\left[1-\exp \left(-\frac{\left(r+S p_{r}\right)^{2}}{2 \Sigma_{11}(S)}\right)\right] \\
& =p_{r}-\frac{4 N r_{e}}{\gamma_{0}} \frac{1}{\sqrt{2 \pi \sigma_{z}^{2}}} e^{-\left(z^{2} / 2 \sigma_{z}^{2}\right)} \int_{-\infty}^{\infty} d S e^{\left(-2 S^{2}+2 z S\right) / \sigma_{z}^{2}} \frac{1}{r+S p_{r}}\left[1-\exp \left(-\frac{\left(r+S p_{r}\right)^{2}}{2 \Sigma_{11}(S)}\right)\right] .
\end{aligned}
$$

The integration cannot be carried out easily, mainly due to the $S$ in the denominator of the exponential. However, if some conditions are met, we can truncate the high power of $S$. For our purpose, we will keep only the terms up to the second order of $S$. The validity of this truncation will be investigated numerically in a later section. To the second order, the integral can be carried out easily,

$$
\begin{gathered}
\int_{-\infty}^{\infty} d S e^{\left(-2 S^{2}+2 z S\right) / \sigma_{z}^{2}}=\sqrt{\frac{\pi \sigma_{z}^{2}}{2}} e^{z^{2} / 2 \sigma_{z}^{2}}, \quad \int_{-\infty}^{\infty} d S e^{\left(-2 S^{2}+2 z S\right) / \sigma_{z}^{2}} S=\frac{z}{2} \sqrt{\frac{\pi \sigma_{z}^{2}}{2}} e^{z^{2} / 2 \sigma_{z}^{2}}, \\
\int_{-\infty}^{\infty} d S e^{\left(-2 S^{2}+2 z S\right) / \sigma_{z}^{2}} S^{2}=\frac{\sigma_{z}^{2}}{4} \sqrt{\frac{\pi \sigma_{z}^{2}}{2}}\left(1+\frac{z^{2}}{\sigma_{z}^{2}}\right) e^{z^{2} / 2 \sigma_{z}^{2}}
\end{gathered}
$$

Note that the factor $e^{z^{2} / 2 \sigma_{z}^{2}}$, which is in all the integrals above, will cancel $e^{-\left(z^{2} / 2 \sigma_{z}^{2}\right)}$ outside the original integral.

To calculate Eq. (48), we need to expand the integrand in $S$, keep up to the second order, and integrate it. To make the writing short and concise, we define

$$
\phi_{f}=\frac{1}{r+S p_{r}}\left[1-\exp \left(-\frac{\left(r+S p_{r}\right)^{2}}{2 \Sigma_{11}(S)}\right)\right] .
$$

We expand the exponential into an infinite series

$$
\begin{aligned}
\phi_{f} & =\frac{1}{r+S p_{r}}\left(1+\sum_{k=0}^{\infty} \frac{\left(-\frac{\left(r+S p_{r}\right)^{2}}{2 \Sigma_{11}(S)}\right)^{k}}{k !}\right) \\
& =-\frac{1}{r+S p_{r}} \sum_{k=1}^{\infty} \frac{\left(-\frac{\left(r+S p_{r}\right)^{2}}{2 \Sigma_{11}(S)}\right)^{k}}{k !} \\
& =-\sum_{k=1}^{\infty} \frac{(-1)^{k}\left(r+S p_{r}\right)^{2 k-1}}{k !} \frac{1}{\left(2 \epsilon_{0} \beta\right)^{k}\left(1-2 \alpha S / \beta+\gamma S^{2} / \beta\right)^{k}} \\
& =-\sum_{k=1}^{\infty} \frac{(-1)^{k}\left(r+S p_{r}\right)^{2 k-1}}{k !} \frac{1}{\left(2 \epsilon_{0} \beta\right)^{k}}\left[\sum_{m=0}^{\infty}\left(\frac{2 \alpha S}{\beta}-\frac{\gamma S^{2}}{\beta}\right)^{m}\right]^{k} .
\end{aligned}
$$

When $r \gg\left|z p_{r}\right|$, with the help of the binomial expansion, we obtain

$$
\left(r+S p_{r}\right)^{n}=r^{n}+n r^{n-1} S p_{r}+\frac{n(n-1)}{2} r^{n-2} S^{2} p_{r}^{2}+o\left(S^{3}\right) .
$$

Now, we expand the sum over $m$ up to $S^{2}$,

$$
\sum_{m=0}^{\infty}\left(\frac{2 \alpha S}{\beta}-\frac{\gamma S^{2}}{\beta}\right)^{m}=1+\frac{2 \alpha S}{\beta}-\frac{\gamma S^{2}}{\beta}+\frac{4 \alpha^{2} S^{2}}{\beta^{2}}+o\left(S^{3}\right),
$$

and take the power $k$ of this. The result is

$$
\left[\sum_{m=0}^{\infty}\left(\frac{2 \alpha S}{\beta}-\frac{\gamma S^{2}}{\beta}\right)^{m}\right]^{k}=1+\frac{2 k \alpha S}{\beta}+\left(-\frac{k \gamma}{\beta}+\frac{4 k \alpha^{2}}{\beta^{2}}+\frac{2 k(k-1) \alpha^{2}}{\beta^{2}}\right) S^{2} .
$$


Combining these two results and keeping only up to the second order in $s$,

$$
\begin{aligned}
\phi_{f}= & -\sum_{k=1}^{\infty} \frac{(-1)^{k}}{\left(2 \epsilon_{0} \beta\right)^{k} k !}\left(r+S p_{r}\right)^{2 k-1}\left[\sum_{m=0}^{\infty}\left(\frac{2 \alpha S}{\beta}-\frac{\gamma S^{2}}{\beta}\right)^{m}\right]^{k} \\
= & -\sum_{k=1}^{\infty} \frac{(-1)^{k}}{\left(2 \epsilon_{0} \beta\right)^{k} k !}\left[r^{2 k-1}+(2 k-1) r^{2 k-2} S p_{r}+(2 k-1)(k-1) r^{2 k-3} S^{2} p_{r}^{2}\right] \\
& \times\left[1+\frac{2 k \alpha S}{\beta}+\left(-\frac{k \gamma}{\beta}+\frac{4 k \alpha^{2}}{\beta^{2}}+\frac{2 k(k-1) \alpha^{2}}{\beta^{2}}\right) S^{2}\right] \\
= & -\sum_{k=1}^{\infty} \frac{(-1)^{k}}{\left(2 \epsilon_{0} \beta\right)^{k} k !} r^{2 k-1}+S\left(\frac{2 \alpha k}{\beta r}+\frac{(2 k-1) p_{r}}{r^{2}}\right) r^{2 k} \\
& +S^{2}\left(\frac{2 \alpha k(2 k-1) p_{r}}{\beta r^{2}}+\frac{2 k(k+1) \alpha^{2}}{\beta^{2} r}+\frac{p_{r}^{2}(2 k-1)(k-1)}{r^{3}}-\frac{\gamma k}{\beta r}\right) r^{2 k} .
\end{aligned}
$$

Before integrating, we should perform summation first. The generic form of the sum can be written as

$$
\sum_{k=1}^{\infty} \frac{(-1)^{k} \psi(k)}{k !\left(2 \epsilon_{0} \beta\right)^{k}}
$$

For $\psi(k)=\xi^{k}$,

$$
\sum_{k=1}^{\infty} \frac{(1)^{k} \xi^{k}}{k !\left(2 \epsilon_{0} \beta\right)^{k}}=\exp \left(-\frac{\xi}{2 \epsilon_{0} \beta}\right)-1
$$

For $\psi(k)=k \xi^{k}$,

$$
\sum_{k=1}^{\infty} \frac{(-1)^{k} k \xi^{k}}{k !\left(2 \epsilon_{0} \beta\right)^{k}}=\sum_{k=1}^{\infty} \frac{-\xi}{2 \epsilon_{0} \beta} \frac{(-1)^{k-1} \xi^{k-1}}{(k-1) !\left(2 \epsilon_{0} \beta\right)^{k-1}} .
$$

Now, resetting the starting point of the index $k$ to 0 ,

$$
\sum_{k=1}^{\infty} \frac{(-1)^{k} k \xi^{k}}{k !\left(2 \epsilon_{0} \beta\right)^{k}}=-\frac{\xi}{2 \epsilon_{0} \beta} \sum_{k=0}^{\infty} \frac{(-1)^{k} \xi^{k}}{k !\left(2 \epsilon_{0} \beta\right)^{k}}=-\frac{\xi}{2 \epsilon_{0} \beta} \exp \left(-\frac{\xi}{2 \epsilon_{0} \beta}\right)
$$

For $\psi(k)=k^{2} \xi^{k}$

$$
\begin{aligned}
\sum_{k=1}^{\infty} \frac{(-1)^{k} k^{2} \xi^{k}}{k !\left(2 \epsilon_{0} \beta\right)^{k}} & =\sum_{k=1}^{\infty} \frac{(-1)^{k}\left[k(k-1) \xi^{k}+k \xi^{k}\right]}{k !\left(2 \epsilon_{0} \beta\right)^{k}} \\
& =\sum_{k=1}^{\infty} \frac{(-1)^{k} k(k-1) \xi^{k}}{k !\left(2 \epsilon_{0} \beta\right)^{k}}+\sum_{k=1}^{\infty} \frac{(-1)^{k} k \xi^{k}}{k !\left(2 \epsilon_{0} \beta\right)^{k}} \\
& =\frac{\xi^{2}}{(2 \epsilon \beta)^{2}} \sum_{k=2}^{\infty} \frac{(-1)^{k-2} \xi^{k-2}}{(k-2) !\left(2 \epsilon_{0} \beta\right)^{k-2}}-\frac{\xi}{2 \epsilon_{0} \beta} \exp \left(-\frac{\xi}{2 \epsilon_{0} \beta}\right) \\
& =\frac{\xi^{2}}{\left(2 \epsilon_{0} \beta\right)^{2}} \exp \left(-\frac{\xi}{2 \epsilon_{0} \beta}\right)-\frac{\xi}{2 \epsilon_{0} \beta} \exp \left(-\frac{\xi}{2 \epsilon_{0} \beta}\right) .
\end{aligned}
$$

With these ingredients, we can sum $\phi_{f}$ over $k$. After a little algebra, we obtain

$$
\begin{gathered}
\phi_{f}=-\frac{1}{r}\left(e^{-\left(r^{2} / 2 \epsilon_{0} \beta\right)}-1\right)-S\left[-\frac{p_{r}}{r^{2}}\left(e^{-\left(r^{2} / 2 \epsilon_{0} \beta\right)}-1\right)+2\left(\frac{\alpha}{\beta r}+\frac{p_{r}}{r^{2}}\right)\left(-\frac{r^{2}}{2 \epsilon_{0} \beta}\right) e^{-\left(r^{2} / 2 \epsilon_{0} \beta\right)}\right] \\
-S^{2}\left[\frac{p_{r}^{2}}{r^{3}}\left(e^{-\left(r^{2} / 2 \epsilon_{0} \beta\right)}-1\right)+\left(-\frac{\gamma}{\beta r}+\frac{2 \alpha^{2}}{\beta^{2} r}-\frac{3 p_{r}^{2}}{r^{3}}-\frac{2 \alpha p_{r}}{\beta r^{2}}\right)\left(-\frac{r^{2}}{2 \epsilon_{0} \beta}\right) e^{-\left(r^{2} / 2 \epsilon_{0} \beta\right)}\right. \\
\left.+\left(\frac{2 \alpha^{2}}{\beta^{2} r}+\frac{2 p_{r}^{2}}{r^{3}}+\frac{4 \alpha p_{r}}{\beta r^{2}}\right)\left(\frac{r^{4}}{\left(2 \epsilon_{0} \beta\right)^{2}}-\frac{r^{2}}{2 \epsilon_{0} \beta}\right) e^{-\left(r^{2} / 2 \epsilon_{0} \beta\right)}\right] .
\end{gathered}
$$


Now, one might ask if some of the denominators will cause singularity at $r=0$. This is not the case. Those singular terms cancel each other and only the positive power of $r$ remains after the expansion of the exponentials. In fact, when $r=0$,

$$
\phi_{f}=\frac{p_{r}}{2 \epsilon_{0} \beta} S-\frac{\alpha p_{r}}{\epsilon_{0} \beta^{2}} S^{2} .
$$

Finally, we can express the original integral $F$, after integration over $S$ with the help of Eq. (49):

$$
\begin{gathered}
F\left(r, p_{r}, z\right)=-\frac{2 N r_{e}}{\gamma_{0}}\left\{\frac{1}{r}\left(e^{-\left(r^{2} / 2 \epsilon_{0} \beta\right)}-1\right)+\frac{z}{2}\left[-\frac{p_{r}}{r^{2}}\left(e^{-\left(r^{2} / 2 \epsilon_{0} \beta\right)}-1\right)+2\left(\frac{\alpha}{\beta r}+\frac{p_{r}}{r^{2}}\right)\left(-\frac{r^{2}}{2 \epsilon_{0} \beta}\right) e^{-\left(r^{2} / 2 \epsilon_{0} \beta\right)}\right]\right. \\
+\frac{\sigma_{z}^{2}+z^{2}}{4}\left[\frac{p_{r}^{2}}{r^{3}}\left(e^{-\left(r^{2} / 2 \epsilon_{0} \beta\right)}-1\right)+\left(-\frac{\gamma}{\beta r}+\frac{2 \alpha^{2}}{\beta^{2} r}-\frac{3 p_{r}^{2}}{r^{3}}-\frac{2 \alpha p_{r}}{\beta r^{2}}\right)\left(-\frac{r^{2}}{2 \epsilon_{0} \beta}\right) e^{-\left(r^{2} / 2 \epsilon_{0} \beta\right)}\right. \\
\left.\left.+\left(\frac{2 \alpha^{2}}{\beta^{2} r}+\frac{2 p_{r}^{2}}{r^{3}}+\frac{4 \alpha p_{r}}{\beta r^{2}}\right)\left(\frac{r^{4}}{\left(2 \epsilon_{0} \beta\right)^{2}}-\frac{r^{2}}{2 \epsilon_{0} \beta}\right) e^{-\left(r^{2} / 2 \epsilon_{0} \beta\right)}\right]\right\}
\end{gathered}
$$

Now, let us do the same for $r$,

$$
r^{\text {new }}=r-\frac{N r_{e}}{\gamma_{0}} \int_{-\infty}^{\infty} \frac{d z_{*}}{\sqrt{2 \pi \sigma_{z}^{2}}} \exp \left(-\frac{z_{*}^{2}}{2 \sigma_{z}^{2}}\right) \frac{2 S}{r+S p_{r}}\left[1-\exp \left(-\frac{\left(r+S p_{r}\right)^{2}}{2 \Sigma_{11}(S)}\right)\right]=r+H .
$$

Compared to the $p_{r}$ case, powers of $S$ are augmented by 1 . Thus,

$$
\begin{aligned}
H=\frac{2 N r_{e}}{\gamma_{0}}\left\{\frac{z}{2 r}\left(e^{-\left(r^{2} / 2 \epsilon_{0} \beta\right)}-1\right)\right. \\
\left.\quad+\frac{\sigma_{z}^{2}+z^{2}}{4}\left[-\frac{p_{r}}{r^{2}}\left(e^{-\left(r^{2} / 2 \epsilon_{0} \beta\right)}-1\right)+2\left(\frac{\alpha}{\beta r}+\frac{p_{r}}{r^{2}}\right)\left(-\frac{r^{2}}{2 \epsilon_{0} \beta}\right) e^{-\left(r^{2} / 2 \epsilon_{0} \beta\right)}\right]\right\} .
\end{aligned}
$$

As we know, we do not have to work on $z$. Hence,

$$
z^{\text {new }}=z
$$

Now, we can finally work on $\epsilon$ :

$$
\epsilon^{\text {new }}=\epsilon-\frac{1}{2} \int f p_{r}+\frac{1}{4}\left(\int f\right)^{2}-\int g .
$$

Since the $f^{2}$ term is $N r_{e} / \gamma$ smaller than any other terms, we simply ignore it.

The second term has already been calculated for $p_{r}$; we just have to multiply the result of $p_{r}$ by $p_{r}$. So let us work on $g$ :

$$
\phi_{g}=\frac{1}{\Sigma_{11}(S)} \frac{d \Sigma_{11}(S)}{d S} \frac{1}{2} \exp \left(-\frac{\left(r+S p_{r}\right)^{2}}{2 \Sigma_{11}(S)}\right)
$$

Calculating the new term up to $S^{2}$,

$$
\frac{1}{\Sigma_{11}(S)} \frac{d \Sigma_{11}(S)}{d S}=\frac{2}{\beta}\left[-\alpha+S\left(\gamma-\frac{2 \alpha^{2}}{\beta}\right)+S^{2}\left(\frac{3 \alpha \gamma}{\beta}-\frac{4 \alpha^{3}}{\beta^{2}}\right)+o\left(S^{3}\right)\right] .
$$

Combining this with the expansion of $\exp \left\{-\left[\left(r+S p_{r}\right)^{2} / 2 \Sigma_{11}(S)\right]\right\}$, we obtain

$$
\begin{aligned}
\phi_{g}=\sum_{k=0}^{\infty} \frac{(-1)^{k}}{k !\left(2 \epsilon_{0} \beta\right)^{k}} \frac{r^{2 k}}{\beta}\{ & -\alpha+S\left[\gamma-\frac{2 \alpha^{2}}{\beta}-\frac{2 \alpha^{2} k}{\beta}-\frac{2 \alpha k p_{r}}{r}\right] \\
& +S^{2}\left[\frac{3 \alpha \gamma}{\beta}-\frac{4 \alpha^{3}}{\beta^{2}}+\frac{3 k \alpha \gamma}{\beta}-\frac{2 k(k+3) \alpha^{3}}{\beta^{2}}-\frac{k(2 k-1) \alpha p_{r}^{2}}{r^{2}}\right] \\
& \left.-\frac{4 \alpha^{2} p_{r}\left(k^{2}+k\right)}{\beta r}+\frac{2 k \gamma p_{r}}{r}\right\} .
\end{aligned}
$$


Now, we perform summation over $k$ using the following formulas: For $\psi(k)=\xi^{k}$,

$$
\sum_{k=0}^{\infty} \frac{(-1)^{k} \xi^{k}}{k !\left(2 \epsilon_{0} \beta\right)^{k}}=\exp \left(-\frac{\xi}{2 \epsilon_{0} \beta}\right) \text {. }
$$

For $\psi(k)=k \xi^{k}$

$$
\sum_{k=0}^{\infty} \frac{(-1)^{k} k \xi^{k}}{k !\left(2 \epsilon_{0} \beta\right)^{k}}=\sum_{k=1}^{\infty} \frac{(-1)^{k} k \xi^{k}}{k !\left(2 \epsilon_{0} \beta\right)^{k}}=-\frac{\xi}{2 \epsilon_{0} \beta} \exp \left(-\frac{\xi}{2 \epsilon_{0} \beta}\right)
$$

For $\psi(k)=k^{2} \xi^{k}$

$$
\sum_{k=0}^{\infty} \frac{(-1)^{k} k^{2} \xi^{k}}{k !\left(2 \epsilon_{0} \beta\right)^{k}}=\sum_{k=1}^{\infty} \frac{(-1)^{k} k^{2} \xi^{k}}{k !\left(2 \epsilon_{0} \beta\right)^{k}}=\frac{\xi^{2}}{\left(2 \epsilon_{0} \beta\right)^{2}} \exp \left(-\frac{\xi}{2 \epsilon_{0} \beta}\right)-\frac{\xi}{2 \epsilon_{0} \beta} \exp \left(-\frac{\xi}{2 \epsilon_{0} \beta}\right) .
$$

Using these summation formulas,

$$
\begin{aligned}
\phi_{g}=\left\{-\alpha e^{-\left(r^{2} / 2 \epsilon_{0} \beta\right)}+S\left[\left(\gamma-\frac{2 \alpha^{2}}{\beta}\right) e^{-\left(r^{2} / 2 \epsilon_{0} \beta\right)}+\left(\frac{\alpha^{2}}{\beta}+\frac{\alpha p_{r}}{r}\right) \frac{r^{2}}{\epsilon_{0} \beta} e^{-\left(r^{2} / 2 \epsilon_{0} \beta\right)}\right]\right. \\
+S^{2}\left[\left(\frac{3 \alpha \gamma}{\beta}-\frac{4 \alpha^{3}}{\beta^{2}}\right) e^{-\left(r^{2} / 2 \epsilon_{0} \beta\right)}-\left(\frac{3 \alpha \gamma}{\beta}-\frac{6 \alpha^{3}}{\beta^{2}}+\frac{\alpha p_{r}^{2}}{r^{2}}-\frac{4 \alpha^{2} p_{r}}{\beta r}+\frac{2 \gamma p_{r}}{r}\right) \frac{r^{2}}{2 \epsilon_{0} \beta} e^{-\left(r^{2} / 2 \epsilon_{0} \beta\right)}\right. \\
\left.\left.-\frac{\alpha r^{2}}{\epsilon_{0} \beta}\left(\frac{\alpha^{2}}{\beta^{2}}+\frac{p_{r}^{2}}{r^{2}}+\frac{2 \alpha p_{r}}{\beta r}\right)\left(\frac{r^{2}}{2 \epsilon_{0} \beta}-1\right) e^{-\left(r^{2} / 2 \epsilon_{0} \beta\right)}\right]\right\} .
\end{aligned}
$$

Thus, integrating this expression, we finally obtain

$$
\begin{aligned}
G=\frac{N r_{e}}{\gamma_{0} \beta}\left\{-\alpha e^{-\left(r^{2} / 2 \epsilon_{0} \beta\right)}+\frac{z}{2}\left[\left(\gamma-\frac{2 \alpha^{2}}{\beta}\right) e^{-\left(r^{2} / 2 \epsilon_{0} \beta\right)}+\left(\frac{\alpha^{2}}{\beta}+\frac{\alpha p_{r}}{r}\right) \frac{r^{2}}{\epsilon_{0} \beta} e^{-\left(r^{2} / 2 \epsilon_{0} \beta\right)}\right]\right. \\
+\frac{\sigma_{z}^{2}+z^{2}}{4}\left[\left(\frac{3 \alpha \gamma}{\beta}-\frac{4 \alpha^{3}}{\beta^{2}}\right) e^{-\left(r^{2} / 2 \epsilon_{0} \beta\right)}-\left(\frac{3 \alpha \gamma}{\beta}-\frac{6 \alpha^{3}}{\beta^{2}}+\frac{\alpha p_{r}^{2}}{r^{2}}-\frac{4 \alpha^{2} p_{r}}{\beta r}+\frac{2 \gamma p_{r}}{r}\right) \frac{r^{2}}{2 \epsilon_{0} \beta} e^{-\left(r^{2} / 2 \epsilon_{0} \beta\right)}\right. \\
\left.\left.-\frac{\alpha r^{2}}{\epsilon_{0} \beta}\left(\frac{\alpha^{2}}{\beta^{2}}+\frac{p_{r}^{2}}{r^{2}}+\frac{2 \alpha p_{r}}{\beta r}\right)\left(\frac{r^{2}}{2 \epsilon_{0} \beta}-1\right) e^{-\left(r^{2} / 2 \epsilon_{0} \beta\right)}\right]\right\}
\end{aligned}
$$

As we mentioned previously, the $r$ in the denominators does not lead to singularity at $r=0$. In fact, for $r=0$, we have

$$
G=\frac{N r_{e}}{\gamma_{0} \beta}\left\{-\alpha+\frac{z}{2}\left(\gamma-\frac{2 \alpha^{2}}{\beta}\right)+\frac{\sigma_{z}^{2}+z^{2}}{4}\left[\left(\frac{3 \alpha \gamma}{\beta}-\frac{4 \alpha^{3}}{\beta^{2}}\right)-\frac{\alpha p_{r}^{2}}{2 \epsilon_{0} \beta}+\frac{\alpha p_{r}^{2}}{\epsilon_{0} \beta}\right]\right\} .
$$

\section{Simulation and conclusion}

To investigate the validity of our formula, a simulation has been carried out. First, particles are distributed in Gaussian distributions according to $\sigma_{r}, \sigma_{z}$, $\sigma_{p r}$, and $\sigma_{e p}$. Then, for each particle, we apply, on the one hand, slice methods [4] using synchro-beam map, where slices are from 1 to 9 , and, on the other hand, the method developed in this paper from zeroth- to second-order approximation. To quantify the accuracy, we calculated "the mean squared deviation" $\left\langle\left(r_{k \text {-slice }}-\right.\right.$ $\left.\left.r_{m \text {-order }}\right)^{2}+\beta^{2}\left(p_{r, k \text {-slice }}-p_{r, m \text {-order }}\right)^{2}\right\rangle$ for each possible combination. The result is tabulated in Table I, and numerical values of the parameters are given in Table II.

First, look at the column marked zeroth order. In this column, the mean squared deviations are calculated for 1slice to 9-slice methods with respect to the zeroth-order approximation. The numbers are relatively large and do not vary from slice to slice. This implies that kicks obtained from the zeroth-order method are far from any synchro-beam map method. Thus, it is not good enough to represent synchro-beam by the zeroth-order approximation, in general.

TABLE I. Calculation of the ensemble average $\left\langle\left(r_{k \text {-slice }}-r_{m \text {-order }}\right)^{2}+\beta^{2}\left(p_{r, k \text {-slice }}-p_{r, m \text {-order }}\right)^{2}\right\rangle$. These discrepancies (dimensions $\mathrm{m}^{2}$ ) are small compared to $\sigma_{r}^{2}=10^{-8} \mathrm{~m}^{2}$.

\begin{tabular}{lccc}
\hline \hline & Zeroth order & First order & Second order \\
\hline 1 slice & $1.59 \times 10^{-12}$ & $1.38 \times 10^{-14}$ & $1.90 \times 10^{-15}$ \\
3 slice & $1.62 \times 10^{-12}$ & $1.56 \times 10^{-14}$ & $1.31 \times 10^{-15}$ \\
5 slice & $1.60 \times 10^{-12}$ & $2.44 \times 10^{-14}$ & $4.53 \times 10^{-16}$ \\
7 slice & $1.60 \times 10^{-12}$ & $2.48 \times 10^{-14}$ & $4.61 \times 10^{-16}$ \\
9 slice & $1.59 \times 10^{-12}$ & $2.48 \times 10^{-14}$ & $4.80 \times 10^{-16}$ \\
\hline \hline
\end{tabular}


TABLE II. Numbers used in a simulation.

\begin{tabular}{cc}
\hline \hline Number of sample particles & 100000 \\
$N$ & $1.0 \times 10^{12}$ \\
$\alpha$ & 0 \\
$\beta(\mathrm{m})$ & 0.2 \\
$\gamma\left(\mathrm{m}^{-1}\right)$ & 5 \\
$\gamma_{0}($ Lorentz factor) & $1.0 \times 10^{4}$ \\
$\sigma_{r}(\mathrm{~m})$ & $1.9 \times 10^{-4}$ \\
$\sigma_{z}(\mathrm{~m})$ & $1.0 \times 10^{-2}$ \\
$\sigma_{p r}$ & $1.3 \times 10^{-3}$ \\
$\sigma_{\epsilon}$ & $6.0 \times 10^{-4}$ \\
$\xi$ (linear beam-beam tune shift) & 0.124 \\
\hline \hline
\end{tabular}

Now, let us move to the next column marked first order. Now, the quantities are substantially smaller; however, the quantity minimizes at the 1 -slice method. This implies that the first-order method is not a bad approximation to synchro-beam map and the first-order method resembles the 1-slice method most. The latter further implies that we should make better approximation to include slice effects. Hence, the first-order approximation is still insufficient to describe the beam-beam interaction, in general.

Finally, we look at the last column, where the quantities are taken with respect to the second-order method. The quantity has been reduced further and now minimizes at the 5-slice method. This implies that the approximation using up to the second order includes not only the benefit of synchro-beam map but also the benefit of slice effects. The latter implication is backed more strongly by the fact that the quantities remain small even with the 7- and 9 -slice methods.

Those who are discontented with the accuracy of the 5 -slice method are encouraged to derive third- or even higher-order corrections to our calculation. Since all the necessary techniques to derive such corrections are shown here, it would be just tedious algebra.

Since $\alpha$ is 0 at IP for many cases, one can simplify the formula derived here considerably and the reduction would further facilitate the derivation of higher-order correction, if necessary.

This new method has the advantage of being a point interaction or a single kick. It is not necessary to propagate back and forth between IP and CPs to apply beam-beam interaction.

\section{ACKNOWLEDGMENT}

I thank my thesis advisor Richard Talman for numerous suggestions and corrections.

[1] S. Krishnagopal and R. Siemann, Phys. Rev. D 41, 2312 (1990).

[2] H. M. Kohji Hirata and F. Ruggiero, Part. Accel. 40, 205 (1993).

[3] R. Talman, in Physics of Particle Accelerators, edited by Melvin Month and Margaret Deines, AIP Conf. Proc. No. 153 (AIP, New York, 1987).

[4] S. Krishnagopal, Ph.D. thesis, Cornell University, 1991. 